\title{
Coimplications in the set of discrete fuzzy numbers
}

\author{
Juan Vicente Riera ${ }^{1}$ Joan Torrens ${ }^{1}$ \\ ${ }^{1}$ University of the Balearic Islands, Palma de Mallorca 07122, Spain
}

\begin{abstract}
Given a coimplication function $J$ defined on the finite chain $L_{n}=\{0, \ldots, n\}$, a method for extending $J$ to the set of discrete fuzzy numbers whose support is an interval contained in $L_{n}$ (denoted by $\left.\mathcal{A}_{1}^{L_{n}}\right)$ is given. The resulting extension is in fact a fuzzy coimplication on $\mathcal{A}_{1}^{L_{n}}$ preserving many of the usual properties of coimplications. In particular, duality between implications and coimplications is preserved.
\end{abstract}

Keywords: coimplication function, implication function, discrete fuzzy number, finite chain.

\section{Introduction}

The study of logical connectives is a key point in the development of the theory of fuzzy sets and fuzzy logic. On one hand, t-norms and tconorms are commonly used to model conjunctions and disjunctions respectively. In this context, tnorms and t-conorms are dual operators one of each other through strong negations, that usually perform complements in the fuzzy sets theory.

In this direction, one of the most important types of connectives in fuzzy logic are fuzzy implication functions. They are widely used in fuzzy control and approximate reasoning, because they are essential in modelling fuzzy conditionals as well as in the inference process. For this reason, implication functions have been extensively studied from both, the applicational and the theoretical points of view (see for instance [1], [18] and the references therein). $\mathrm{Cu}-$ riously, with respect to their dual operators, called co-implications, there are only some few works dealing with them (see $[8,11,21,24,22,26]$ ).

When we deal with fuzzy logic the used scale is always the unit interval $[0,1]$. However, in many applications only a finite number of values is used and even only qualitative information is handled. For this reason, many authors have studied in last years operations defined on a finite chain $L_{n}$, usually called discrete operations. For instance, tnorms and t-conorms were characterized in [20], uninorms and nullnorms in [14], idempotent uninorms in [9], a non-commutative version of nullnorms in [10], weighted means in [13], smooth aggregation functions in [17], copulas in [19] and also implications functions in [15] and [16]. It is proved in [20] that only the number of elements of the finite chain $L_{n}$ is relevant when we deal with monotonic operations on $L_{n}$, and so the finite chain used in many of the mentioned works is the most simple one $L_{n}=\{0,1, \ldots, n\}$.

The representative finite chain $L_{n}$ is usually considered to model linguistic hedges in qualitative information, which is often interpreted to take values in a totally ordered finite scale like this:

$$
\mathcal{L}=\{\text { Extremely Bad, Very Bad, Bad, Fair, Good }
$$

$$
\text { Very Good, Extremely Good\}. }
$$

However, the modelling of linguistic information is limited because the information provided by experts for each variable must be expressed by a simple linguistic term. In most cases, this is a problem for experts because their opinion does not agree with a concrete term. On the contrary, experts' values are usually expressions like "better than Good", "between Fair and Very Good" or even more complex expressions.

To avoid the limitation above (see $[5,6,25])$ the authors deal with the possibility of extending monotonic operations on $L_{n}$ to operations on the set of discrete fuzzy numbers whose support is an interval contained in $L_{n}$, usually denoted by $\mathcal{A}_{1}^{L_{n}}$. The idea lies on the fact that any discrete fuzzy number $A \in \mathcal{A}_{1}^{L_{n}}$ can be considered (identifying the scale $\mathcal{L}$ given in (1) with $L_{n}$ with $n=6$ ) as an assignment of a $[0,1]$-value to each term in our linguistic scale. As an example, the above mentioned expression "between Fair and Very Good" can be performed, for instance, by a discrete fuzzy number $A \in \mathcal{A}_{1}^{L_{6}}$, with support given by the subinterval $[F, V G]$ (that corresponds to the interval $[3,5]$ in $\left.L_{6}\right)$. The values of $A$ in its support should be described by experts, allowing in this way a complete flexibility of the qualitative valuation. Thus, operations on $\mathcal{A}_{1}^{L_{n}}$ allow us to manage qualitative information in a more flexible way. In [5] t-norms and t-conorms on $\mathcal{A}_{1}^{L_{n}}$ are described and studied, as well as it is done for uninorms, nullnorms and general aggregation functions in [25]. In both cases, an example of application in decision making or subjective evaluation is included.

Following in this direction we want to study coimplication functions on $\mathcal{A}_{1}^{L_{n}}$. We present a method of constructing such coimplications from discrete coimplications defined on $L_{n}$, and we prove that many of the usual properties of coimplications are preserved by this extension method. 


\section{Preliminaries}

We will suppose the reader to be familiar with the basic theory of logical connectives in both, the fuzzy and the discrete cases. For operators on $[0,1]$ see [12] and for operators on finite scales see [20] and also [15]. Thus, we recall only some key definitions and results for the rest of the paper.

\subsection{Discrete coimplications}

Let $(\mathcal{P}, \leq)$ be a bounded ordered set with smallest element 0 and greatest element $n$.

Definition 2.1 [8] A coimplication function $J$ on $(\mathcal{P}, \leq)$ is a binary operator $J: \mathcal{P} \times \mathcal{P} \rightarrow \mathcal{P}$ that is nonincreasing in the first variable, nondecreasing in the second one and satisfies the corner conditions $J(0,0)=0, J(n, n)=0$ and $J(0, n)=n$.

Remark 2.2 [8] Note that for any coimplication function $J$ on $(\mathcal{P}, \leq)$ it turns out that

$$
J(n, \alpha)=J(\alpha, 0)=0, \text { for all } \alpha \in \mathcal{P}
$$

a property called the absorption principle.

Definition 2.3 [8] A border coimplication $J$ on $(\mathcal{P}, \leq)$ is a coimplication function that satisfies the neutrality principle

$$
J(0, \beta)=\beta, \text { for all } \beta \in \mathcal{P} .
$$

Definition 2.4 [8] Let $J$ be a coimplication function on $(\mathcal{P}, \leq)$. Then $J$ satisfies

i) the exchange principle $(E P)$, if

$$
J(x, J(y, z))=J(y, J(x, z)) \text { for all } x, y, z \in \mathcal{P}
$$

ii) the contraposition law $(C L)$ with respect to the strong negation $N$ on $\mathcal{P}$, if

$$
J(N(y), N(x))=J(x, y) \text { for all } x, y \in \mathcal{P}
$$

iii) A model coimplication $J$ is a border coimplication that is contrapositive and satisfies the exchange principle.

A particular case of bounded ordered set is when we consider the finite chain $L_{n}=\{0, \ldots, n\} \subset \mathbb{N}$. Operators defined on $L_{n}$ are usually called discrete operations and they have been studied by many authors (see $[7,13,14,20]$ ). In these studies the following condition, generally used as a discrete counterpart of continuity, is considered.

Definition 2.5 A function $f: L_{n} \rightarrow L_{n}$ is said to be smooth if it satisfies: $|f(x)-f(x-1)| \leq 1$ for all $x \in L_{n}$ with $x \geq 1$.

Definition 2.6 A binary operation $F: L_{n}^{2} \rightarrow L_{n}$ is said to be smooth when each one of its vertical and horizontal sections $(F(x,$.$) and F(., y)$, respectively) are smooth.
Smooth discrete t-norms and t-conorms were characterized in [20]. There, it is also proved that there is one and only one strong negation on $L_{n}$ which is given by

$$
N(x)=n-x \quad \text { for all } \quad x \in L_{n} .
$$

The four most usual ways to define implication functions on $L_{n}$ were investigated in [15] and [16]. That is,

$$
\begin{aligned}
& I(x, y)=\max \left\{z \in L_{n} \mid T(x, z) \leq y\right\} \text { (R-implications) } \\
& I(x, y)=S(N(x), y) \text { (S-implications) } \\
& I(x, y)=S(N(x), T(x, y)) \in L_{n} \text { (QL-operations) } \\
& I(x, y)=S(T(N(x), N(y)), y) \in L_{n} \text { (D-operations) }
\end{aligned}
$$

for all $x, y \in L_{n}$, where $T$ is a (smooth) t-norm, $S$ a (smooth) t-conorm and $N$ the strong negation on $L_{n}$ given by equation (2). From now on, $N$ will always denote such a negation.

On the other hand, co-implications on finite scales, were introduced in [24]. In particular, strong coimplications derived from smooth t-norms and residual co-implications derived from smooth tconorms were studied in detail. The structure of such co-implications were given and several properties were investigated.

\subsection{Discrete fuzzy numbers}

In this section, we recall some definitions and the main results about discrete fuzzy numbers which will be used later. By a fuzzy subset of $\mathbb{R}$, we mean a function $A: \mathbb{R} \rightarrow[0,1]$. For each fuzzy subset $A$, let $A^{\alpha}=\{x \in \mathbb{R}: A(x) \geq \alpha\}$ for any $\alpha \in(0,1]$ be its $\alpha$-level set (or $\alpha$-cut). By $\operatorname{supp}(A)$, we mean the support of $A$, i.e. the set $\{x \in \mathbb{R}: A(x)>0\}$. By $A^{0}$, we mean the closure of $\operatorname{supp}(A)$.

Definition 2.7 [27] A fuzzy subset $A$ of $\mathbb{R}$ with membership mapping $A: \mathbb{R} \rightarrow[0,1]$ is called discrete fuzzy number if its support is finite, i.e., there exist $x_{1}, \ldots, x_{n} \in \mathbb{R}$ with $x_{1}<x_{2}<\ldots<x_{n}$ such that $\operatorname{supp}(A)=\left\{x_{1}, \ldots, x_{n}\right\}$, and there are natural numbers $s, t$ with $1 \leq s \leq t \leq n$ such that:

1. $A\left(x_{i}\right)=1$ for any natural number $i$ with $s \leq i \leq$ $t$ (core)

2. $A\left(x_{i}\right) \leq A\left(x_{j}\right)$ for each natural number $i, j$ with $1 \leq i \leq j \leq s$

3. $A\left(x_{i}\right) \geq A\left(x_{j}\right)$ for each natural number $i, j$ with $t \leq i \leq j \leq n$

Remark 2.8 If the fuzzy subset $A$ is a discrete fuzzy number then the support of $A$ coincides with its closure, i.e. $\operatorname{supp}(A)=A^{0}$.

From now on, we will denote the set of discrete fuzzy numbers by $D F N$ and the abbreviation $d f n$ will denote a discrete fuzzy number.

Theorem 2.9 [28] (Representation of discrete fuzzy numbers) Let $A$ be a discrete fuzzy number. Then the following statements (1)-(4) hold: 
1. $A^{\alpha}$ is a nonempty finite subset of $\mathbb{R}$, for any $\alpha \in[0,1]$

2. $A^{\alpha_{2}} \subseteq A^{\alpha_{1}}$ for any $\alpha_{1}, \alpha_{2} \in[0,1]$ with $0 \leq$ $\alpha_{1} \leq \alpha_{2} \leq 1$

3. For any $\alpha_{1}, \alpha_{2} \in[0,1]$ with $0 \leq \alpha_{1} \leq \alpha_{2} \leq 1$, if $x \in A^{\alpha_{1}}-A^{\alpha_{2}}$ we have $x<y$ for all $y \in A^{\alpha_{2}}$, or $x>y$ for all $y \in A^{\alpha_{2}}$

4. For any $\alpha_{0} \in(0,1]$, there exists a real number $\alpha_{0}^{\prime}$ with $0<\alpha_{0}^{\prime}<\alpha_{0}$ such that $A^{\alpha_{0}^{\prime}}=A^{\alpha_{0}}$ i.e. $A^{\alpha}=A^{\alpha_{0}}$ for any $\left.\alpha \in\left[\alpha_{0}^{\prime}, \alpha_{0}\right]\right)$.

Conversely, if for any $\alpha \in[0,1]$, there exists $A^{\alpha} \subset$ $\mathbb{R}$ satisfying the conditions (1)-(4) stated above, then there exists a unique $A \in D F N$ such that its $\alpha$-cuts are exactly the sets $A^{\alpha}$ for any $\alpha \in[0,1]$.

$>$ From now on, we will denote by $\mathcal{A}_{1}^{L_{n}}$ the set of discrete fuzzy numbers whose support is an interval contained in the finite chain $L_{n}$.

Let $A, B \in \mathcal{A}_{1}^{L_{n}}$ be two discrete fuzzy numbers, and let $A^{\alpha}=\left[x_{1}^{\alpha}, x_{p}^{\alpha}\right], B^{\alpha}=\left[y_{1}^{\alpha}, y_{k}^{\alpha}\right]$ be its $\alpha$-level cuts for $A$ and $B$ respectively.

For each $\alpha \in[0,1]$, we consider the following sets,

$$
\begin{array}{r}
\min (A, B)^{\alpha}= \\
\left\{z \in L_{n} \mid \min \left(x_{1}^{\alpha}, y_{1}^{\alpha}\right) \leq z \leq \min \left(x_{p}^{\alpha}, y_{k}^{\alpha}\right)\right\} \text { and } \\
\max (A, B)^{\alpha}= \\
\left\{z \in L_{n} \mid \max \left(x_{1}^{\alpha}, y_{1}^{\alpha}\right) \leq z \leq \max \left(x_{p}^{\alpha}, y_{k}^{\alpha}\right)\right\}
\end{array}
$$

Proposition 2.10 [2] There exist two unique discrete fuzzy numbers, that we will denote by $\operatorname{MIN}(A, B)$ and $M A X(A, B)$, such that they have the sets $\min (A, B)^{\alpha}$ and $\max (A, B)^{\alpha}$ as $\alpha$-cuts respectively.

The following result holds for $\mathcal{A}_{1}^{L_{n}}$, but is not true for the set of discrete fuzzy numbers in general(see [3]).

Theorem 2.11 [3]

The triplet $\left(\mathcal{A}_{1}^{L_{n}}, M I N, M A X\right)$ is a bounded distributive lattice where $1_{n} \in \mathcal{A}_{1}^{L_{n}}$ (the unique discrete fuzzy number whose support is the singleton $\{n\}$ ) and $1_{0} \in \mathcal{A}_{1}^{L_{n}}$ (the unique discrete fuzzy number whose support is the singleton $\{0\}$ ) are the maximum and the minimum, respectively.

Remark 2.12 [3] Using these operations, we can define a partial order on $\mathcal{A}_{1}^{L_{n}}$ in the usual way: $A \preceq B$ if and only if $M I N(A, B)=A$, or equivalently, $A \preceq B$ if and only if $M A X(A, B)=B$ for any $A, B \in \mathcal{A}_{1}^{L_{n}}$. Equivalently, we can also define the partial ordering in terms of $\alpha$-cuts:

\section{$A \preceq B$ if and only if $\min \left(A^{\alpha}, B^{\alpha}\right)=A^{\alpha}$}

$A \preceq B$ if and only if $\max \left(A^{\alpha}, B^{\alpha}\right)=B^{\alpha}$

Any t-norm and t-conorm on $L_{n}$ was extended to a t-norm and a t-conorm on $\mathcal{A}_{1}^{L_{n}}$ in [5].

Theorem 2.13 [5] Let $T(S)$ be a t-norm (tconorm) on $L_{n}$ and let

$$
\begin{aligned}
\mathcal{T}(\mathcal{S}): \mathcal{A}_{1}^{L_{n}} \times \mathcal{A}_{1}^{L_{n}} & \rightarrow \mathcal{A}_{1}^{L_{n}} \\
(A, B) & \longmapsto \mathcal{T}(\mathcal{S})(A, B)
\end{aligned}
$$

be the extension of $t$-norm (t-conorm) $T(S)$ to $\mathcal{A}_{1}^{L_{n}}$, defined as follows: $\mathcal{T}(\mathcal{S})(A, B)$ is the discrete fuzzy number whose $\alpha$-cuts are the sets

$$
\begin{gathered}
\left\{z \in L_{n} \mid T(S)\left(\min A^{\alpha}, \min B^{\alpha}\right) \leq z \leq\right. \\
\left.T(S)\left(\max A^{\alpha}, \max B^{\alpha}\right)\right\}
\end{gathered}
$$

for each $\alpha \in[0,1]$. Then, $\mathcal{T}(\mathcal{S})$ is a t-norm $(t$ conorm) on the bounded set $\mathcal{A}_{1}^{L_{n}}$.

Similarly to Theorem 2.13 above, it is possible to extend the unique strong negation function $N$ defined on $L_{n}$ to a strong negation function $\mathcal{N}$ on the bounded lattice $\mathcal{A}_{1}^{L_{n}}$.

Proposition 2.14 [4] Let us consider the strong negation $N$ on the finite chain $L_{n}=\{0,1, \cdots, n\}$. The mapping

$$
\begin{aligned}
\mathcal{N}: \mathcal{A}_{1}^{L_{n}} & \longrightarrow \mathcal{A}_{1}^{L_{n}} \\
A & \mapsto \mathcal{N}(A)
\end{aligned}
$$

is a strong negation on the bounded distributive lattice $\mathcal{A}_{1}^{L_{n}}=\left(\mathcal{A}_{1}^{L_{n}}, M I N, M A X\right)$ where $\mathcal{N}(A)$ is the discrete fuzzy number whose $\alpha$-level sets are

$$
\mathcal{N}(A)^{\alpha}=\left[N\left(x_{p}^{\alpha}\right), N\left(x_{1}^{\alpha}\right)\right]
$$

for each $\alpha \in[0,1]$ (being $A^{\alpha}=\left[x_{1}^{\alpha}, x_{p}^{\alpha}\right]$ the $\alpha$-cuts of $A$ ).

\section{Coimplication functions on $\mathcal{A}_{1}^{L_{n}}$}

In this section we wish to study if it is possible to build coimplication functions on the bounded set $\mathcal{A}_{1}^{L_{n}}$ by extending a discrete coimplication function $J$ defined on the finite chain $L_{n}$. Let us begin with some notation. If

$$
\begin{aligned}
O: L_{n} \times L_{n} & \longrightarrow L_{n} \\
(x, y) & \longmapsto O(x, y)
\end{aligned}
$$

is a binary discrete function on $L_{n}$ (e.g. a t-norm, t-conorm, implication, coimplication, etc.), we will denote as well by $O$, the binary operation

$$
\begin{aligned}
O: 2^{L_{n}} \times 2^{L_{n}} & \longrightarrow 2^{L_{n}} \\
(\mathrm{X}, \mathrm{Y}) & \longmapsto O(\mathrm{X}, \mathrm{Y})
\end{aligned}
$$

where $O(\mathrm{X}, \mathrm{Y})=\{O(x, y) \mid x \in \mathrm{X}, y \in \mathrm{Y}\}$.

Lemma 3.1 Let us consider $A, B \in \mathcal{A}_{1}^{L_{n}}$ and let $J$ be a discrete coimplication function on the finite chain $L_{n}$. Then the following equalities

$$
\begin{aligned}
\min J\left(A^{\alpha}, B^{\alpha}\right) & =J\left(\max A^{\alpha}, \min B^{\alpha}\right) \\
\max J\left(A^{\alpha}, B^{\alpha}\right) & =J\left(\min A^{\alpha}, \max B^{\alpha}\right)
\end{aligned}
$$

hold for each $\alpha \in[0,1]$, where $A^{\alpha}, B^{\alpha}$ are the $\alpha$-cut sets for $A$ and $B$ respectively. 
Proof We only show the second relation because the proof of the first one is similar. Note that the inequality

$$
J\left(\min A^{\alpha}, \max B^{\alpha}\right) \leq \max J\left(A^{\alpha}, B^{\alpha}\right)
$$

is clear. To show the converse inequality, since $J$ is decreasing in the first variable and increasing in the second one, we have

$$
J(x, y) \leq J\left(\min A^{\alpha}, \max B^{\alpha}\right)
$$

for all $x \in A^{\alpha}$ and for all $y \in B^{\alpha}$. Thus

$$
\max J\left(A^{\alpha}, B^{\alpha}\right) \leq J\left(\min A^{\alpha}, \max B^{\alpha}\right) .
$$

Proposition 3.2 Let us consider $A, B \in \mathcal{A}_{1}^{L_{n}}$ and let $J$ be any discrete coimplication function on the finite chain $L_{n}$. There exists a unique discrete fuzzy number whose $\alpha$-cuts are exactly the sets

$$
\left\{z \in L_{n} \mid \min J\left(A^{\alpha}, B^{\alpha}\right) \leq z \leq \max J\left(A^{\alpha}, B^{\alpha}\right)\right\}
$$

that will be denoted by $\mathcal{J}(A, B)$. Moreover, $\mathcal{J}(A, B) \in \mathcal{A}_{1}^{L_{n}}$.

Proof We will see that the sets $C^{\alpha}$ (defined according to expression (4)) satisfy the four conditions of Theorem 2.9:

1. For each $\alpha \in[0,1], C^{\alpha}$ is a nonempty finite set, because of $A^{\alpha}$ and $B^{\alpha}$ are both nonempty finite sets (the discrete fuzzy numbers are normal fuzzy subsets).

2. $C^{\beta} \subseteq C^{\alpha}$ for any $\alpha, \beta \in[0,1]$ with $0 \leq \alpha \leq$ $\beta \leq 1$.

Because if $A, B \in \mathcal{A}_{1}^{L_{n}}$ we know that

$$
\begin{aligned}
A^{\beta} \subseteq A^{\alpha} \text { implies } & \min A^{\alpha} \leq \min A^{\beta} \\
\text { and } & \max A^{\beta} \leq \max A^{\alpha} \\
B^{\beta} \subseteq B^{\alpha} \text { implies } & \min B^{\alpha} \leq \min B^{\beta} \\
\text { and } & \max B^{\beta} \leq \max B^{\alpha}
\end{aligned}
$$

Moreover, as $J$ is decreasing in the first variable, using the relation (6) we obtain

$$
J\left(\max A^{\alpha}, z\right) \leq J\left(\max A^{\beta}, z\right) \text { for all } z \in L_{n}
$$

And, in particular by relation (7),

$$
J\left(\max A^{\alpha}, \min B^{\alpha}\right) \leq J\left(\max A^{\beta}, \min B^{\beta}\right)
$$

Thus, from Lemma 3.1

$$
\min J\left(A^{\alpha}, B^{\alpha}\right) \leq \min J\left(A^{\beta}, B^{\beta}\right)
$$

Similarly, we can see that

$$
\max J\left(A^{\beta}, B^{\beta}\right) \leq \min J\left(A^{\alpha}, B^{\alpha}\right) .
$$

Combining the previous conditions (9) and (10), we obtain $C^{\beta}=\left\{z \in L_{n}\right.$ $\left.\min J\left(A^{\beta}, B^{\beta}\right) \leq z \leq \max J\left(A^{\beta}, B^{\beta}\right)\right\} \subseteq\{z \in$ $\left.L_{n} \mid \min J\left(A^{\alpha}, B^{\alpha}\right) \leq z \leq \max J\left(A^{\alpha}, B^{\alpha}\right)\right\}=$ $C^{\alpha}$.

Therefore, $C^{\beta} \subseteq C^{\alpha}$.
3. If $x \in C^{\alpha}-C^{\beta}$ then $x \in L_{n}$ and $x$ does not belong to $C^{\beta}$, hence either $x<$ $J\left(\max A^{\beta}, \min B^{\beta}\right)$, which is the minimum of $C^{\beta}$, or $x>J\left(\min A^{\beta}, \max B^{\beta}\right)$, which is the maximum of $C^{\beta}$.

4. As $A, B \in \mathcal{A}_{1}^{L_{n}}$, according to Theorem 2.9, for each $\alpha \in(0,1]$ there exist real numbers $\alpha_{1}^{\prime}$ and $\alpha_{2}^{\prime}$ with $0<\alpha_{1}^{\prime}<\alpha$ and $0<\alpha_{2}^{\prime}<\alpha$ such that for each $r \in\left[\alpha_{1}^{\prime}, \alpha\right], A^{\alpha}=A^{r}$. Moreover $B^{\alpha}=$ $B^{r}$, for each $r \in\left[\alpha_{2}^{\prime}, \alpha\right]$. Thus, if $\alpha^{\prime}=\alpha_{1}^{\prime} \vee \alpha_{2}^{\prime}$, we can obtain:

$$
\begin{aligned}
& \min A^{r}=\min A^{\alpha} \text { and } \max A^{r}=\max A^{\alpha} \\
& \min B^{r}=\min B^{\alpha} \text { and } \max B^{r}=\max B^{\alpha}
\end{aligned}
$$

for each $r \in\left[\alpha^{\prime}, \alpha\right]$. Therefore

$$
\begin{aligned}
& J\left(\max A^{r}, \min B^{r}\right)=J\left(\max A^{\alpha}, \min B^{\alpha}\right) \\
& J\left(\min A^{r}, \max B^{r}\right)=J\left(\min A^{\alpha}, \max B^{\alpha}\right) .
\end{aligned}
$$

Hence, by Lemma 3.1

$$
\begin{aligned}
\min J\left(A^{r}, B^{r}\right) & =\min J\left(A^{\alpha}, B^{\alpha}\right) \\
\max J\left(A^{r}, B^{r}\right) & =\max J\left(A^{\alpha}, B^{\alpha}\right)
\end{aligned}
$$

and so,

$C^{r}=\left\{z \in L_{n} \mid \min J\left(A^{r}, B^{r}\right) \leq z \leq\right.$ $\left.\max J\left(A^{r}, B^{r}\right)\right\}=\left\{z \in L_{n} \mid \min J\left(A^{\alpha}, B^{\alpha}\right) \leq\right.$ $\left.z \leq \max J\left(A^{\alpha}, B^{\alpha}\right)\right\}=C^{\alpha}$ for each $r \in\left[\alpha^{\prime}, \alpha\right]$.

As the sets $C^{\alpha}$ fulfill for each $\alpha \in[0,1]$ the conditions stated in Theorem 2.9, there exists a unique discrete fuzzy number, that will be denoted by $\mathcal{J}(A, B)$, such that its $\alpha$-cuts are exactly these sets.

In addition, from the construction of the sets $C^{\alpha}$ for each $\alpha \in[0,1]$, it is clear that the sets $C^{\alpha}$ are intervals contained in the finite chain $L_{n}$. Thus, $\mathcal{J}(A, B) \in \mathcal{A}_{1}^{L_{n}}$.

The previous proposition will allow us to define a binary operation $\mathcal{J}$ on $\mathcal{A}_{1}^{L_{n}}$ from a coimplication function $J$ defined on the finite chain $L_{n}$.

Definition 3.3 Let us consider a coimplication function $J$ on the finite chain $L_{n}$. The binary operation on $\mathcal{A}_{1}^{L_{n}}$ defined as follows

$$
\begin{array}{r}
\mathcal{J}: \mathcal{A}_{1}^{L_{n}} \times \mathcal{A}_{1}^{L_{n}} \longrightarrow \mathcal{A}_{1}^{L_{n}} \\
(A, B) \longmapsto \mathcal{J}(A, B)
\end{array}
$$

will be called the extension of the discrete coimplication function $J$ to $\mathcal{A}_{1}^{L_{n}}$, being $\mathcal{J}(A, B)$ the discrete fuzzy number whose $\alpha$-cuts are the sets

$$
\left\{z \in L_{n} \mid \min J\left(A^{\alpha}, B^{\alpha}\right) \leq z \leq \max J\left(A^{\alpha}, B^{\alpha}\right)\right\}
$$

for each $\alpha \in[0,1]$.

Now we wish to study if the extension of the discrete coimplication function $J$ to $\mathcal{A}_{1}^{L_{n}}$ defined above satisfies the condition to be a coimplication function on the bounded set $\mathcal{A}_{1}^{L_{n}}$ according to Definition 2.1. 
Theorem 3.4 Let $J$ be a coimplication function on $L_{n}$. Then the extension of the discrete coimplication function $J$ to $\mathcal{A}_{1}^{L_{n}}$ is a coimplication function on $\mathcal{A}_{1}^{L_{n}}$.

Proof To show this result, we will prove all the conditions stated in Definition 2.1. Let us consider $A, B \in \mathcal{A}_{1}^{L_{n}}$.

- We wish to see that $\mathcal{J}$ is decreasing in the first variable, i.e. if $A \preceq B$ then $\mathcal{J}(A, C) \succeq$ $\mathcal{J}(B, C)$ for all $C \in \mathcal{A}_{1}^{L_{n}}$. According to Remark 2.12, it is equivalent to prove that $\min \left(\mathcal{J}(B, C)^{\alpha}, \mathcal{J}(A, C)^{\alpha}\right)=\mathcal{J}(B, C)^{\alpha}$ for all $\alpha \in[0,1]$. As $A \preceq B$ then $\min A^{\alpha} \leq \min B^{\alpha}$ and $\max A^{\alpha} \leq \max B^{\alpha}$. Thus as $J$ is a coimplication function on $L_{n}$, it is a decreasing function in the first variable, then by Lemma 3.1

$$
\begin{aligned}
\min J\left(A^{\alpha}, C^{\alpha}\right) & =J\left(\max A^{\alpha}, \min C^{\alpha}\right) \\
& \geq J\left(\max B^{\alpha}, \min C^{\alpha}\right) \\
& =\min J\left(B^{\alpha}, C^{\alpha}\right)
\end{aligned}
$$

Analogously,

$$
\begin{aligned}
\max J\left(A^{\alpha}, C^{\alpha}\right) & =J\left(\min A^{\alpha}, \max C^{\alpha}\right) \\
& \geq J\left(\min B^{\alpha}, \max C^{\alpha}\right) \\
& =\max J\left(B^{\alpha}, C^{\alpha}\right)
\end{aligned}
$$

Finally using the relations (11) and (12) above $\mathcal{J}(A, C)^{\alpha}=\left\{z \in L_{n} \mid \min J\left(A^{\alpha}, C^{\alpha}\right) \leq z \leq\right.$ $\left.\max J\left(A^{\alpha}, C^{\alpha}\right)\right\} \supseteq\left\{z \in L_{n} \mid \min J\left(B^{\alpha}, C^{\alpha}\right) \leq\right.$ $\left.z \leq \max J\left(B^{\alpha}, C^{\alpha}\right)\right\}=\mathcal{J}(B, C)^{\alpha}$

for each $\alpha \in[0,1]$.

- The increasingness with the second variable follows similarly.

- With respect to the boundary conditions we have $\mathcal{J}\left(1_{0}, 1_{0}\right)^{\alpha}=\left\{z \in L_{n} \mid \min J\left(1_{0}{ }^{\alpha}, 1_{0}{ }^{\alpha}\right) \leq z \leq\right.$ $\left.\max J\left(1_{0}{ }^{\alpha}, 1_{0}{ }^{\alpha}\right)\right\}=\left\{z \in L_{n} \mid 0 \leq z \leq 0\right\}=$ $\{0\}=1_{0}{ }^{\alpha}$ for all $\alpha \in[0,1]$.

Then $\mathcal{J}\left(1_{0}, 1_{0}\right)=1_{0}$.

The other two conditions $\mathcal{J}\left(1_{n}, 1_{n}\right)=1_{0}$ and $\mathcal{J}\left(1_{0}, 1_{n}\right)=1_{n}$ follows similarly.

Remark 3.5 Note that, since $\mathcal{J}$ is a coimplication on $\mathcal{A}_{1}^{L_{n}}$, it must also satisfy the absorption property, that is,

for all $A \in \mathcal{A}_{1}^{L_{n}}$.

$$
\mathcal{J}\left(1_{n}, A\right)=\mathcal{J}\left(A, 1_{0}\right)=1_{0}
$$

Example 3.6 Let us consider the chain $L_{6}$ and

$$
\begin{aligned}
A & =\{0.3 / 0,0.5 / 1,1 / 2,0.8 / 3,0.5 / 4\} \\
B & =\{0.6 / 2,0.8 / 3,0.9 / 4,1 / 5,0.8 / 6\}
\end{aligned}
$$

belonging to $\mathcal{A}_{1}^{L_{6}}$. If we consider the Eukasiewicz coimplication function

$$
J_{L}(x, y)=\left\{\begin{array}{lll}
y-x & \text { if } & y \geq x \\
0 & \text { if } & x>y
\end{array}\right.
$$

and the Kleene-Dienes coimplication in $L_{6}$

$$
J(x, y)=\min (6-x, y),
$$

we obtain that

$$
\begin{aligned}
\mathcal{J}_{\mathcal{L}}(A, B) & =\{0.8 / 0,0.8 / 1,0.9 / 2,1 / 3,0.8 / 4,0.5 / 5\} \\
\mathcal{J}(A, B) & =\{0.6 / 2,0.8 / 3,1 / 4,0.5 / 5\}
\end{aligned}
$$

In the next two propositions we deal with some other properties that are many times required for coimplications, depending on the context.

Proposition 3.7 If a coimplication function $J$ is a border coimplication on $L_{n}$ then the coimplication $\mathcal{J}$ generated by $J$ according to Definition 3.3 is a border coimplication on the bounded set $\mathcal{A}_{1}^{L_{n}}$ too.

Proof To show this condition it is enough to see that $\mathcal{J}\left(1_{0}, B\right)^{\alpha}=$

$$
\begin{array}{r}
\left\{z \in L_{n} \mid \min J\left(1_{0}{ }^{\alpha}, B^{\alpha}\right) \leq z \leq \max J\left(1_{0}{ }^{\alpha}, B^{\alpha}\right)\right\} \\
=\left\{z \in L_{n} \mid J\left(0, \min B^{\alpha}\right) \leq z \leq J\left(0, \max B^{\alpha}\right)\right\} \\
=\left\{z \in L_{n} \mid \min B^{\alpha} \leq z \leq \max B^{\alpha}\right\} \\
=B^{\alpha} \text { for each } \alpha \in[0,1] .
\end{array}
$$

Proposition 3.8 Let $J$ be a coimplication function on $L_{n}$ and $\mathcal{J}$ its extension on $\mathcal{A}_{1}^{L_{n}}$.

i) $J$ satisfies $(E P)$ if and only if $\mathcal{J}$ satisfies $(E P)$.

ii) $J$ satisfies $(C L)$ with respect to $N$ if and only if $\mathcal{J}$ satisfies $(C L)$ with respect to $\mathcal{N}$.

Proof Now, we will study each case.

i) Suppose that $J$ satisfies $(E P)$ and consider $A, B, C \in \mathcal{A}_{1}^{L_{n}}$. It is enough to show that

$$
\mathcal{J}(A, \mathcal{J}(B, C))^{\alpha}=\mathcal{J}(B, \mathcal{J}(A, C))^{\alpha},
$$

or equivalently, is equivalent to show these two conditions

$$
\min J\left(A^{\alpha}, \mathcal{J}(B, C)^{\alpha}\right)=\min J\left(B^{\alpha}, \mathcal{J}(A, C)^{\alpha}\right)
$$

$\max J\left(A^{\alpha}, \mathcal{J}(B, C)^{\alpha}\right)=\max J\left(B^{\alpha}, \mathcal{J}(A, C)^{\alpha}\right)$

However, applying Lemma 3.1 the minimums in the condition (13) above are given by $J\left(\max A^{\alpha}, J\left(\max B^{\alpha}, \min C^{\alpha}\right)\right)$ and $J\left(\max B^{\alpha}, J\left(\max A^{\alpha}, \min C^{\alpha}\right)\right), \quad$ respectively, which coincide because $J$ satisfies $(E P)$. Analogously, it is possible to see the equality (14).

Conversely, suppose that $\mathcal{J}$ satisfies $(E P)$ and consider $a, b, c \in L_{n}$. Let $1_{a}, 1_{b}$ and $1_{c}$ the discrete fuzzy numbers whose support is given by the singletons $\{a\},\{b\},\{c\}$, respectively. Then clearly

$$
\mathcal{J}\left(1_{a}, \mathcal{J}\left(1_{b}, 1_{c}\right)\right)=\mathcal{J}\left(1_{b}, \mathcal{J}\left(1_{a}, 1_{c}\right)\right)
$$

and this implies directly that $J$ satisfies $(E P)$. 
ii) Similarly as above, for all $\alpha \in[0,1]$ and $A, B \in$ $\mathcal{A}_{1}^{L_{n}}$ we have

$$
\begin{aligned}
\min J\left(\mathcal{N}(B)^{\alpha}, \mathcal{N}(A)^{\alpha}\right) & =\min J\left(A^{\alpha}, B^{\alpha}\right) \\
\max J\left(\mathcal{N}(B)^{\alpha}, \mathcal{N}(A)^{\alpha}\right) & =\max J\left(A^{\alpha}, B^{\alpha}\right)
\end{aligned}
$$

and then

$$
\mathcal{J}(\mathcal{N}(B), \mathcal{N}(A))^{\alpha}=\mathcal{J}(A, B)^{\alpha}
$$

for all $\alpha \in[0,1]$.

The converse is again similar to the previous step.

Corollary $3.9 \mathrm{~J}$ is a model coimplication on $L_{n}$ if and only if $\mathcal{J}$ is a model coimplication on $\mathcal{A}_{1}^{L_{n}}$.

Fuzzy negations can be build from fuzzy implications, as well as from fuzzy coimplications $[8,1]$. This is a result general in any partially ordered set. Consequently, in $\mathcal{A}_{1}^{L_{n}}$ we have:

Proposition 3.10 Let $\mathcal{J}$ a coimplication on $\mathcal{A}_{1}^{L_{n}}$ obtained from the coimplication $J$ on $L_{n}$. Then the function

$$
\begin{aligned}
\mathcal{N}_{\mathcal{J}}: \mathcal{A}_{1}^{L_{n}} & \longrightarrow \mathcal{A}_{1}^{L_{n}} \\
A & \mapsto \mathcal{N}_{\mathcal{J}}(A)=\mathcal{J}\left(A, 1_{n}\right),
\end{aligned}
$$

is a fuzzy negation on $\mathcal{A}_{1}^{L_{n}}$.

It is well known that the comparison of coimplication functions defined on a finite chain $L_{n}$ is done in the usual way, i.e., pointwise. In the same way, if we consider two coimplications functions $\mathcal{J}_{1}, \mathcal{J}_{2}$ on $\mathcal{A}_{1}^{L_{n}}$ generated by two coimplications $J_{1}, J_{2}$ on $L_{n}$ with $J_{1} \leq J_{2}$ it is possible to obtain a similar order between $\mathcal{J}_{1}$ and $\mathcal{J}_{2}$ as follows.

Proposition 3.11 Let $J_{1}$ and $J_{2}$ be two coimplications functions on the finite chain $L_{n}=$ $\{0,1, \cdots, n\}$ verifying $J_{1}(x, y) \leq J_{2}(x, y)$ for all $(x, y) \in L_{n} \times L_{n}$. Let us consider their extensions on $\mathcal{A}_{1}^{L_{n}}, \mathcal{J}_{1}$ and $\mathcal{J}_{2}$ for $J_{1}$ and $J_{2}$ respectively. Then $\mathcal{J}_{1}(A, B) \preceq \mathcal{J}_{2}(A, B)$ for all $A, B \in \mathcal{A}_{1}^{L_{n}}$.

Proof It is enough to prove that (see Remark 2.12) $\min \left(\mathcal{J}_{1}(A, B)^{\alpha}, \mathcal{J}_{2}(A, B)^{\alpha}\right)=\mathcal{J}_{1}(A, B)^{\alpha}$ for each $\alpha \in[0,1]$. To do it, note that

$$
\begin{aligned}
\min \mathcal{J}_{1}(A, B)^{\alpha} & =\min J_{1}\left(A^{\alpha}, B^{\alpha}\right) \\
& =J_{1}\left(\max A^{\alpha}, \min B^{\alpha}\right) \\
& \leq J_{2}\left(\max A^{\alpha}, \min B^{\alpha}\right) \\
& =\min J_{2}\left(A^{\alpha}, B^{\alpha}\right),
\end{aligned}
$$

and similarly for the maximums. This already implies

$$
\min \left(\mathcal{J}_{1}(A, B)^{\alpha}, \mathcal{J}_{2}(A, B)^{\alpha}\right)=\mathcal{J}_{1}(A, B)^{\alpha} .
$$

It is well known that implications and coimplications are dual operators one of each other (see for instance [8]). In our framework this duality can be written as follows.
Proposition 3.12 Let $\mathcal{I}$ be a binary operator on $\mathcal{A}_{1}^{L_{n}}$. Then $\mathcal{I}$ is an implication if and only if its $\mathcal{N}$-dual, $\mathcal{I}^{\mathcal{N}}$, given by

$$
\mathcal{I}^{\mathcal{N}}(A, B)=\mathcal{N}(\mathcal{I}(\mathcal{N}(A), \mathcal{N}(A)))
$$

for all $A, B \in \mathcal{A}_{1}^{L_{n}}$, is a coimplication on $\mathcal{A}_{1}^{L_{n}}$. Similarly, a binary operator $\mathcal{J}$ on $\mathcal{A}_{1}^{L_{n}}$ is a coimplication if and only if its $\mathcal{N}$-dual, $\mathcal{J}^{\mathcal{N}}$ is an implication.

Note that a method for extending discrete implications on $L_{n}$ to implications on $\mathcal{A}_{1}^{L_{n}}$ was given in [23]. In this way we have the following easy result.

Proposition 3.13 Let $I: L_{n}^{2} \rightarrow L_{n}$ be a discrete implication on $L_{n}$ and let $\mathcal{I}$ be its extension to $\mathcal{A}_{1}^{L_{n}}$. Let $I^{*}$ be the $N$-dual coimplication of $I$ and $\mathcal{I}^{*}$ its extension to $\mathcal{A}_{1}^{L_{n}}$. Then it holds $\mathcal{I}^{*}=\mathcal{I}^{\mathcal{N}}$, where $\mathcal{N}$ is the extension of $N$ to $\mathcal{A}_{1}^{L_{n}}$.

The next proposition shows that it is possible to construct a coimplication function on $\mathcal{A}_{1}^{L_{n}}$ from a t-norm $\mathcal{T}$ (the extension of a t-norm $T$ defined on the finite chain $L_{n}$ ) and the strong negation $\mathcal{N}$ (the extension of the strong negation $N$ on $L_{n}$ ). Specifically,

Proposition 3.14 Let $T$ be a t-norm on $L_{n}$ and let $\mathcal{T}$ and $\mathcal{N}$ be the extensions of $T$ and $N$ to $\mathcal{A}_{1}^{L_{n}}$, respectively. Let $\mathcal{J}_{\mathcal{T}, \mathcal{N}}$ be the mapping given by

$$
\begin{aligned}
& \mathcal{J}_{\mathcal{T}, \mathcal{N}}: \mathcal{A}_{1}^{L_{n}} \times \mathcal{A}_{1}^{L_{n}} \longrightarrow \mathcal{A}_{1}^{L_{n}} \\
& (A, B) \quad \mapsto \quad \mathcal{J}_{\mathcal{T}, \mathcal{N}}=\mathcal{T}(\mathcal{N}(A), B),
\end{aligned}
$$

where $\mathcal{T}(\mathcal{N}(A), B)$ is the discrete fuzzy number whose $\alpha$-cuts are the interval of the finite chain $L_{n}$ due by

$\left[T\left(N\left(\max A^{\alpha}\right), \min B^{\alpha}\right), T\left(N\left(\min A^{\alpha}\right), \max B^{\alpha}\right)\right]$

for each $\alpha \in[0,1]$. Then $\mathcal{J}_{\mathcal{T}, \mathcal{N}}$ is a coimplication function on $\mathcal{A}_{1}^{L_{n}}$, that will be called $\mathcal{T}$-coimplication generated from $\mathcal{T}$ and $\mathcal{N}$.

Now, we want to see that any of these Tcoimplications are, in fact, the extension of a $\mathrm{T}$ coimplication on $L_{n}$ using the general method presented in this paper (see Definition 3.3) to build a coimplication function on $\mathcal{A}_{1}^{L_{n}}$.

Proposition 3.15 Let $T$ be a t-conorm on $L_{n}$ and $J$ the discrete $T$-coimplication on $L_{n}$ defined from $T$ and $N$. Let $\mathcal{T}, \mathcal{N}$ and $\mathcal{J}$ be the extensions of $T$, $N$ and $J$ to $\mathcal{A}_{1}^{L_{n}}$, respectively. Then $\mathcal{J}=\mathcal{J}_{\mathcal{T}, \mathcal{N}}$.

Proof Let us consider $A, B \in \mathcal{A}_{1}^{L_{n}}$ and $A^{\alpha}, B^{\alpha}$ their $\alpha$-cuts for each $\alpha \in[0,1]$ respectively. Thus, we have $\mathcal{J}(A, B)^{\alpha}=\left\{z \in L_{n} \mid\right.$ $\left.J\left(\max A^{\alpha}, \min B^{\alpha}\right) \leq z \leq J\left(\min A^{\alpha}, \max B^{\alpha}\right)\right\}$. As $J(a, b)=T(N(a), b)$ for all $a, b \in L_{n}$ we obtain $\mathcal{J}(A, B)^{\alpha}=\left\{z \in L_{n} \mid T\left(N\left(\max A^{\alpha}\right), \min B^{\alpha}\right) \leq\right.$ $\left.z \leq T\left(N\left(\min A^{\alpha}\right), \max B^{\alpha}\right)\right\}=\mathcal{T}(\mathcal{N}(A), B)^{\alpha}=$ $\mathcal{J}_{\mathcal{T}, \mathcal{N}}(A, B)^{\alpha}$ for each $\alpha \in[0,1]$. 


\section{Conclusions}

In this work we have proposed a method to construct coimplication functions defined on the bounded distributive lattice $\mathcal{A}_{1}^{L_{n}}$ from coimplication functions defined on a finite chain $L_{n}$. Also, we investigate some properties of these operators such as boundary properties, the exchange principle or the contraposition law. Moreover, we also prove that duality between implications and coimplications is preserved and so, the $\mathcal{N}$-dual of the extension of a discrete implication on $L_{n}$ coincides with the extension of the corresponding $N$-dual coimplication. Finally, we deal with the special case of $\mathcal{T}$-coimplication generates from a t-norm $\mathcal{T}$ (which is an extension of t-norm $T$ on $L_{n}$ ) and the strong negation $\mathcal{N}$ (extension of the unique strong negation function $N$ on $L_{n}$ ).

\section{Acknowledgments}

This work has been partially supported by the MTM2009-10962 and MTM2009-10320 project grants, both with FEDER support.

\section{References}

[1] M. Baczyński and B. Jayaram, Fuzzy Implications, Studies in Fuzziness and Soft Computing, vol. 231, Springer, Berlin Heidelberg, 2008.

[2] J. Casasnovas and J.V. Riera, Maximum and Mininum of Discrete Fuzzy numbers. In C. Angulo and L. Godo, editors, proceedings of the $10^{\text {th }}$ international conference of the ACIA (CCIA' 07) (Sant Julia de Lòria (Andorra) 2007), Frontiers in Artificial Intelligence and Applications: artificial intelligence research and development 163, pages 273-280, IOS Press, 2007.

[3] J. Casasnovas and J.V. Riera, Lattice properties of discrete fuzzy numbers under extended min and max. In proceedings IFSA-EUSFLAT, pages 647-652, July, Lisbon (Portugal), 2009.

[4] J. Casasnovas and J.V. Riera, S-implications in the set of discrete fuzzy numbers. In proceedings IEEE-WCCI 2010, pages 2741-2747, July, Barcelona (Spain), 2010.

[5] J. Casasnovas and J.V. Riera, Extension of discrete t-norms and t-conorms to discrete fuzzy numbers, Fuzzy Sets and Systems, 167(1):65-81, Elsevier, 2011.

[6] J. Casasnovas and J.V. Riera, Weighted means of subjective evaluations. In R. Seising and V. Sanz, editors, Soft Computing in Humanities and Social Sciences, pages 323-345, SpringerVerlag, Berlin. 2012.

[7] B. De Baets, Model implicators and their characterization. In N. Sreele, editor, proceedings of the First ICSC International Symposium on
Fuzzy logic, (ICSC 1995), pages A42-A49, Academic Press, 1995.

[8] B. De Baets, Coimplicators, the forgotten connectives, Tatra Mountains Mathematical Publications, 12: 229-240, Tatra Mt. Math. Publ., 1997.

[9] B. De Baets, J. Fodor, D. Ruiz-Aguilera and J. Torrens, Idempotent uninorms on finite ordinal scales, International Journal of Uncertainty, Fuzziness and Knowledge-Based Systems, 17:114, World Scientific, 2009.

[10] J.C. Fodor, Smooth associative operations on finite ordinal scales, IEEE Trans. on Fuzzy Systems, 8: 791-795, IEEE Computational Intelligence Society, 2000.

[11] J. Fodor and M. Roubens, Fuzzy preference modelling and multicriteria decision support. In series: System Theory, Knowledge Engineering and Problem Solving 14, Kluwer Academic Publishers, 1994.

[12] E. P. Klement, R. Mesiar and E. Pap, editors. Triangular Norms, in the collection: Trends in Logic - Studia Logica Library vol. 8, Kluwer Academic Publishers, 2000.

[13] A. Kolesarova, G. Mayor and R. Mesiar, Weighted ordinal means, Information Sciences, 177: 3822-3830, Elsevier, 2007.

[14] M. Mas, G. Mayor and J. Torrens, t-operators and uninorms on a finite totally ordered set, International Journal of Intelligent Systems, 14: 909-922, Wiley Company, 1999.

[15] M. Mas, M. Monserrat and J. Torrens, Simplications and R-implications on a finite chain, Kybernetica, 40: 3-20, ÚTIA, 2004.

[16] M. Mas, M. Monserrat and J. Torrens, On two types of discretes implications, International Journal of Approximate Resoning, 40: 262-279, Elsevier, 2005.

[17] M. Mas, M. Monserrat and J. Torrens, Smooth aggregation functions on finite scales. In E. Hullermeier, T. Kruse and F. Hoffmann, editors, proceedings of $13^{\text {th }}$ international Conference on Information Processing and Management of Uncertainty (IPMU 2010), Lecture Notes in Artificial Intelligence 6178, pages 398-407, SpringerVerlag, 2010.

[18] M. Mas, M. Monserrat, J. Torrens and E. Trillas, A survey on Fuzzy Implications Functions, IEEE Transactions on fuzzy systems, 15: $1107-$ 1121, IEEE Computational Intelligence Society, 2007.

[19] G. Mayor, J. Suñer and J. Torrens, Copula-like operations on finite settings, IEEE Transactions on Fuzzy Systems, 13: 468-477, IEEE Computational Intelligence Society, 2005.

[20] G. Mayor and J. Torrens, Triangular norms on discrete settings. In E.P. Klement and R. Mesiar, editors, Logical, Algebraic, Analytic, and Probabilistic Aspects of Triangular Norms, Elsevier, Netherlands, pages 189-230, 2005. 
[21] K. Oh and A. Kandel, Coimplication and its applications to fuzzy expert systems, Information Sciences 56: 247-260, Elsevier, 1991.

[22] R. Reixer and B. Bedregal, Automorphisms acting on N-dual fuzzy functions: implications and coimplications, Anais do CNMAC, 3:229235, SBMAC, 2010.

[23] J. V. Riera and J. Torrens, Fuzzy implications defined on the set of discrete fuzzy numbers. In proceedings of the rth conference of the European Society for Fuzzy Logic and Technology (EUSFLAT-2011), pages 259-266, July 18-22, Aix-les-Bains (France), 2011.

[24] J. V. Riera and Joan Torrens, Coimplications on finite scales. In S. Greco, B. BouchonMeunier, G. Coletti, M. Fedrizzi, B. Matarazzo and R. R. Yager, editors, proceedings of the $14^{\text {th }}$ International Conference on Information Processing and Management of Uncertainty in Knowledge-Based Systems(IPMU 2012), Advances in computational intelligence 298 , pages 325-334, Springer-Verlag, 2012.

[25] J. V. Riera and J. Torrens, Aggregation of subjective evaluations based on discrete fuzzy numbers, Fuzzy Sets and Systems, 191:21-40, Elsevier, 2012.

[26] D. Ruiz and J. Torrens, Residual implications and co-implications from idempotent uninorms, Kybernetika 40: 21-38, ÚTIA, 2004.

[27] W. Voxman, Canonical representations of discrete fuzzy numbers, Fuzzy Sets and Systems, 54: 457-466, Elsevier, 2001.

[28] G. Wang, C. Wu and C. Zhao, Representation and Operations of discrete fuzzy numbers. Southeast Asian Bulletin of Mathematics, 28: 1003-1010, SEAMS, 2005. 